\title{
Téoros
}

Revue de recherche en tourisme

\section{Système d'évaluation des retombées économiques du tourisme au Canada}

\section{Peter Chau}

Volume 7, numéro 3, novembre 1988

Économie du tourisme

URI : https://id.erudit.org/iderudit/1080380ar

DOI : https://doi.org/10.7202/1080380ar

Aller au sommaire du numéro

Éditeur(s)

Université du Québec à Montréal

ISSN

0712-8657 (imprimé)

1923-2705 (numérique)

Découvrir la revue

Citer cet article

Chau, P. (1988). Système d'évaluation des retombées économiques du tourisme au Canada. Téoros, 7(3), 18-23. https://doi.org/10.7202/1080380ar d'utilisation que vous pouvez consulter en ligne.

https://apropos.erudit.org/fr/usagers/politique-dutilisation/ 


\section{Historique}

Au début des années 1970 , les gouvernements fédéral et provinciaux se sont concentrés sur les retombés Economiques qui résulteraient d'un accroissement des activités de l'industrie du tourisme. Parallèlement à cette résolution d'exploiter le potentiel touristique, on s'est rendu compte de l'existence d'un grand nombre de contraintes économiques, sociales et environnementales, et des conséquences de la réalisation ou de l'anéantissement de ce potentiel. Les planificateurs et les décideurs chargés de surveiller l'évolution du tourisme au Canada se sont alors aperçus qu'il leur fallait mettre au point un cadre de travail et des outils appropriés leur permettant de suivre et d'anticiper les tendances du secteur et d'evaluer des projets touristiques, logiquement et systématiquement.

Au cours de l'automne 1973, aidé par le Bureau des conseillers en gestion, 1'Office du Tourisme du Canada mit sur pied une equipe multidisciplinaire de spécialistes chargés d'enquêter à la fois sur les questions fondamentales qui touchent l'avenir du tourisme au Canada et sur les possibilités d'élaborer des modèles et des techniques applicables au tourisme.

L'enquête recommandait principalement de crór des modèles analytiques susceptibles de répondre aux besoins de deux types distincts d'usagers: ceux qui s'intéressent aux problèmes structuraux de l'industrie et aux questions touchant le développement, la planification et la commercialisation ả long terme, - par exemple, faut-il encourager le tourisme dans certaines régions au cours de la prochaine décennie -, et ceux qui s'intéressent a la stratégie à court terme et aux problèmes de tous les jours en matière de planification et de commercialisation. Les parties suivantes de cet article seront consacrées au modele nommé Système d'évaluation des retombées économiques du tourisme au Canada (SERETC, mieux connu sous le nom de modèle des dépenses du tourisme/modèle d'impact du tourisme (MDT/MIT) ${ }^{11}$.

Notions et définitions de base Les études des retombées économiques du tourisme ont pour but de mesurer et d'evaluer l'ampleur et l'importance de la contribution du tourisme à une économie donnée en fonction de la production brute (ventes totales), du revenu (salaires, traitements et bénéfices), de l'emploi, des recettes publiques (taxes, tarifs, droits, redevances, etc.) et de l'investissement des entreprises. Ces études peuvent être menées de façon ponctuelle et porter, par exemple, sur les retombees Economiques du tourisme sur l'emploi dans le secteur de l'hébergement, ou bien de façon globale, c'est-à-dire en simulant systématiquement les dépenses touristiques en vue d'en tirer des données sur les retombées au chapitre du revenu direct et indirect, de l'emploi, des recettes publiques et de l'investissement des entreprises.

\section{Types de retombées économiques}

Une étude globale de retombées économiques du tourisme devrait tenir compte a la fois des retombées directes ou primaires et des retombeses indirectes et induites ou secondaires. Les retombées directes concernent les effets directs de l'activité touristique: par exemple les ventes, les salaires et les traitements et les profits des employés et des propriétaires d'hótels accueillant des touristes, les recettes et les taxes de vente sur les repas consommés au restaurant par des touristes.

Les retombées indirectes concernent les effets de dépenses subséquentes effectuées par les fournisseurs de biens et services en vue de répondre à la demande originale du touriste. Le propriétaire d'un hôtel dépense donc une partie de ses recettes pour s'approvisionner en produits alimentaires et autres chez les fournisseurs locaux qui, à leur tour, achètent des ingrédients, du matériel et des services à d'autres fournisseurs.

Un autre type de retombées secondaires, les retombées induites, représente $l^{\text {'incidence }}$ des salaires et traitements directement gênerés par l'activité touristique sur les biens et services de la région. Les retombées induites devraient aussi inclure les dépenses publiques provoquées par les recettes fiscales entraînées par les dépenses touristiques.

\section{Les dépenses touristiques par rapport aux retombées}

Pour mesurer les retombées économiques du tourisme, il faut d'une part procéder à une estimation du nombre de visiteurs et des dépenses qu'ils effectuent et, d'autre part, evaluer les retombées des recettes touristiques ainsi estimées sur l'économie.

Malheureusement, une grande majorité des études économiques sur le tourisme visent uniquement à évaluer les dépenses touristi-
"Monsieur Peter Chau, gestionnaire, Programme de la Industrie, Sciences et Technologie Canada. 
ques dans une région. Les résultats de ces tudes sont, par conséquent, incomplets et parfois très trompeurs. Il est concevable, par exemple, qu'un groupe de touristes séjournent dans une région sans entrainer aucunes retombées économiques pour cette région. Ils voyageraient sur un avion appartenant dune compagnie d'aviation étrangère, logeraient dans un hôtel appartenant à des étrangers dont le personnel serait composé de nonrésidents et consommeraient des aliments importés.

Par conséquent, bien qu'il soit important d'obtenir des estimations des dépenses touristiques pour mener des études sur les retombées économiques, il faut tenir compte des autres facteurs si l'on veut obtenir des données significatives concernant l'augmentation du revenu, de l'emploi, des recettes publiques et de l'investissement des entreprises.

\section{Méthodes servant à évaluer les retombées économiques du tourisme}

On peut mesurer les retombées économiques du tourisme sur une économie donnée au moyen des trois méthodes suivantes:

\section{1- méthode de l'enquête}

\section{2- méthode de simulation}

\section{3- méthode combinée}

\section{Méthode de l'enquête}

On peut évaluer les retombées économiques du tourisme en se fondant sur les données recueillies au cours d'enquétes sur le tourisme et d'enquêtes non reliées au tourisme. Les enquétes sur le tourisme sont menées auprès de voyageurs, d'entrepreneurs touristiques et de représentants gouvernementaux. Quant aux enquêtes non reliées au tourisme, il s'agit entre autres d'études sur les dépenses du ménage et/ou sur la population active.

En interrogeant les voyageurs à leur domicile, aux frontières, sur les lieux touristiques, par téléphone ou par la poste, et en se servant des techniques d'échantillonnage statistique pour pondérer les résultats, on peut obtenir le volume total et les tendances des dépenses touristiques dans une région donnée. Bien que la qualité et les coûts de cette méthode varient d'enquête à enquéte, les résultats sont en général peu utiles, coûteux et de qualité médiocre ${ }^{(2)}$. Par exemple, l'enquéte sur les voyages des Canadiens (EVC) de 1988 (la meilleure du genre), aura coûté aux Canadiens plus d'un million de dollars. La planification de cette enquête a duré plus de huit mois, bien que l'étude ait déjà eu lieu par le passé, et les résultats annuels ne seront connus que vers la fin de 1989. Cette enquête se sert de la base de sondage existante de l'Enquête sur la population active et bénéficie de l'appon de personnel expérimenté à la fois de Tourisme Canada et de Statistique Canada. La méme EVC, menée séparément, coûterait plus de 4 millions de dollars. Malgré tout cet arsenal technique et professionnel, la qualité des donnees sur les dépenses recueillies par l'EVC reste. statistiquement incertaine, cela à cause des nombreuses faiblesses inhérentes à la méthodologie de ce type d'enquéte.

Même si l'on peut obtenir des données exactes sur les dépenses touristiques au moyen d'enquétes sur le tourisme, des renseignements additionnels provenant d'exploitants et de représentants gouvernementaux sont nécessaires pour pouvoir estimer les retombées économiques sur le plan des ventes, des revenus, de l'emploi, des taxes et de l'investissement des entreprises.

Les enquêtes à grande échelle menées auprès des exploitants et des représentants gouvernementaux sont coûteuses et produisent rarement des estimations utilisables pour un secteur aussi étendu que le tourisme. principalement parce que les entrepreneurs touristiques ne conservent pas dans leurs dossiers des données détaillées. Les entrepreneurs et les représentants gouvernementaux concernés ne sont pas en mesure de ventiler les ventes, les dépenses, les stocks, les taxes, la contribution de la main-d'oeuvre, etc., par groupe de clients (par exemple résidents et non-résidents, touristes étrangers et touristes locaux $)^{(3,4)}$.

De plus, bien qu'il soit théoriquement faisable pour les employés ou des groupes d'employés d'inscrire le temps consacré à servir des touristes ainsi que le montant des ventes et des taxes de ventes payées par ces demiers, ce système serait très coúteux et difficile à mettre en pratique.

En ce qui concerne les sources de données secondaires, celles-ci ne sont disponibles que sous forme très condensée et ne contiennent pas de renseignements sur les retombes 6 conomiques dés dépenses touristiques.

Enfin, aucune des méthodes d'enquêttes précitées ne produit des données utiles sur les effets secondaires du tourisme.

\section{Modèle de simulation}

Pour mesurer les retombées économiques du tourisme au moyen d'un modèle de simulation, il faut mettre sur pied des modèles exprimant les relations de base qui entrent en jeu dans l'univers à l'étude. Par conséquent, pour pouvoir estimer les dépenses touristiques dans une région donnée, il faudrait établir un modèle exprimant les relations de base qui sous-tendent l'univers du voyageur ou le marché touristique de cette région: qui sont les voyageurs (caractéristiques sociodémographiques)? D'où viennent-ils (origine)? Quelles sont leurs habitudes de voyage (moyens de transport, type d'hébergement, de dépenses, durée de séjour, taille du groupe de touristes, distance parcourue, activités entreprises, etc.)? Pourquoi voyagent-ils (but, motivation, etc.)? De même, une étude des retombées économiques du tourisme sur l'économie nationale nécessiterait la mise sur pied d"un modele représentant les structures et les relations de base présentes dans l'économie; le mouvement de production entre secteurs, la consommation, les besoins en capital et en main-d'oeuvre, etc.. Une fois que les relations de base concernant l'univers ont été établies, on peut simuler les effets des changements enregistrés par n'importe lequel de ses éléments en faisant fonctionner le modèle.

\section{Méthode combinée}

L'estimation des retombées économiques du tourisme se fait en deux étapes: l'estimation des dépenses touristiques et l'évaluation des retombées économiques générées par ces dépenses. Bien qu"il soit essentiellement possible de déterminer, au moyen des méthodes d'enquête, l'ensemble des effets économiques de chaque dollar dépensé par un touriste, une telle entreprise serait très peu pratique et excessivement coùteuse. A l'autre extrême, les retombées économiques du tourisme peuvent être obtenues au moyen de modèles de simulation mathématique sans utiliser aucune donnée tirée d'enquétes. Les résultats toutefois seraient considérés comme des hypothèses qu'il faudrait valider. La méthode la plus courante est celle qui se situe entre ces deux extrêmes: il s'agit de se servir de certaines données d'enquêtes pour simuler les données sur les dépenses touristiques qui, à leur tour, serviront à simuler les données sur les retombées économiques.

\section{La méthode canadienne}

Reconnaissant que la collecte de données sur les dépenses touristiques au moyen d'enquêtes sur le tourisme a) est très coủteuse; b) est longue à planifier et à exécuter et tarde à donner des résultats; c) est désagréable pour les répondants, et d) produit des données douteuses sur les dépenses touristiques, et que des données fiables sur l'importance du tourisme pour l'économie sont nécessaires pour prendre des décisions éclairées, Tourisme Canada a demandé au Bureau des conseillers en gestion vers la fin de 1973 de mettre au point la méthodologie la moins couteuse permettant d'estimer les dépenses touristiques et les retombées économiques qui en découlent.

C'est ainsi que fut elaboré le système d'évaluation des retombées économiques du tourisme au Canada (SERETC), qui est le principal élément du système macro-économique intégré du tourisme au Canada, l'autre élément étant le système intégré de prévisions touristiques. Ce document traite uniquement. du système d'évaluation des retombées économiques du tourisme au Canada, qu'on appelle souvent le MDT/MIT (modèle des dépenses du tourisme/modèle de l'impact du tourisme). Les relations entre les divers éléments du système macro-économique intégré du tourisme au Canada figurent au graphique no 1 . 


\section{GRAPHIQUE 1}

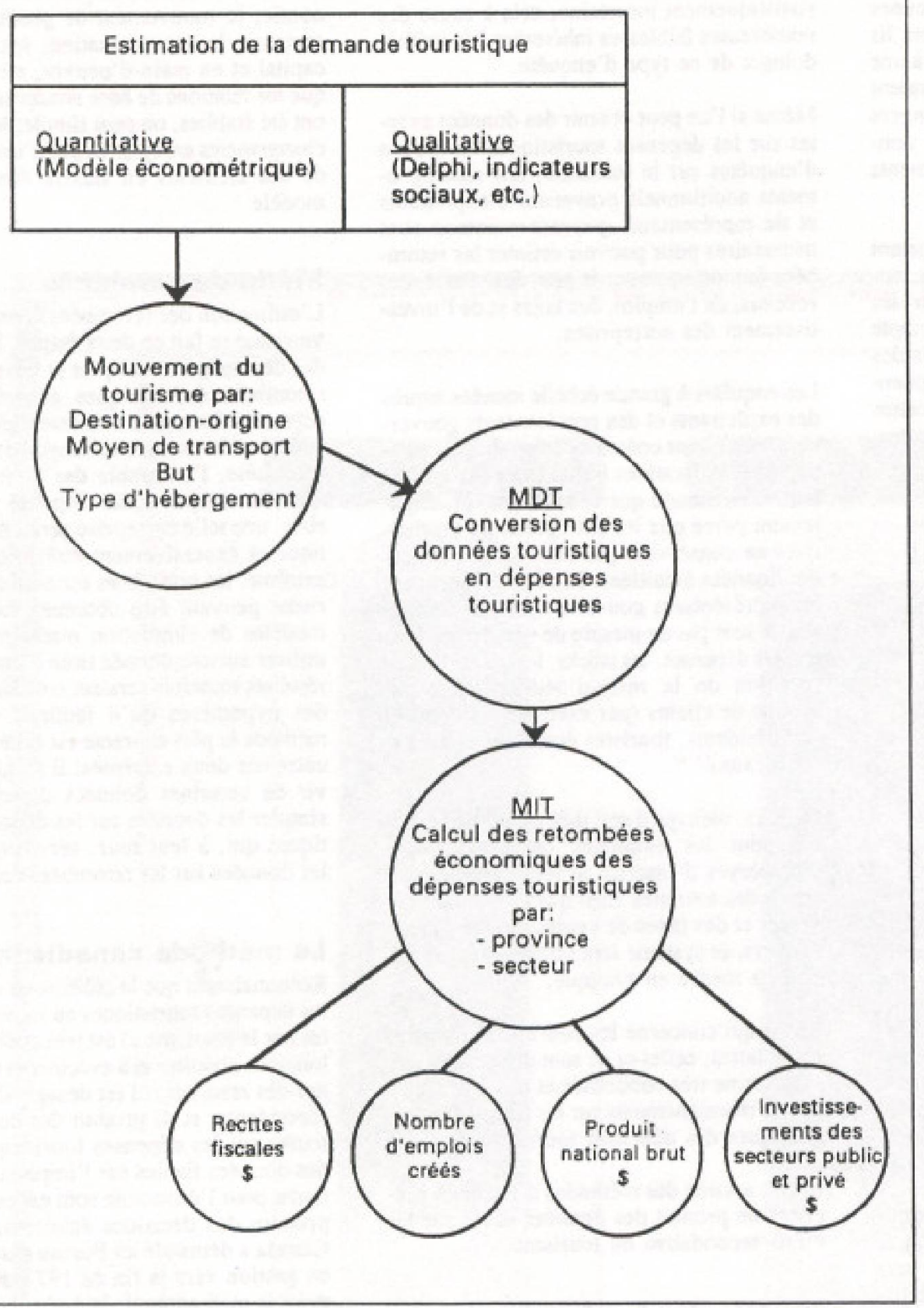

\section{Le système d'évaluation des retombées économiques du tourisme au Canada (SERETC)}

Le principal objectif du SERETC est d'évaluer les retombées économiques des dépenses touristiques. La méthode utilisće consiste à estimer les dépenses touristiques au moyen du MDT et à partir des résultats obtenus, ả calculer les retombées économiques au moyen du MIT sous forme de ventes, revenus, emplois, investissements et recettes publiques.

\section{Modèle d'évaluation des dépenses touristiques (MDT)}

La plus importante fonction du MDT est de produire des données sur les dépenses tou-
Cependant, du point de vue du calcul, le MDT est un systeme très complexe qui exprime les relations de base entrant en jeu dans le secteur de l'hébergement. Le MDT présente une caractéristique novatrice en ce qu'il fournit des estimations sur le nombre total de journées de sejour au moyen des données secondaires existantes tirées des données du secteur de l'hébergement contenant les renseignements essentiels suivants:

a) Tous les établissements commerciaux d'hebergement pour touristes

- par type

- par province

- par région

b) Pour chaque établissement d'hébergement

- le nombre total de chambres

- la date de l'ouverture et de la fermeture de l'exploitation

- les taux d'occupation

- le nombre moyen de personnes par chambre occupé

- les tarifs

- ventes totales

c) Matrices des prix moyens concernant les dépenses touristiques non relićes au logement (par exemple, coût moyen de la nourriture par personne par jour, coût moyen de l'essence par mille, coût moyen des diverses dépenses touristiques par voyage).

La structure du MDT peut être exprimée par une série d'équations mathématiques décrivant les relations entre les trois variables et à l'intérieur de celles-ci, " $i$ ", " "j" ", et " $k$ "; " $\mathrm{i}$ " représentant le type d'hébergement, " $\mathrm{j}$ " les régions de la province et " $\mathrm{k}$ " le mois de $1^{t}$ année ${ }^{(6)}$.

Les quatre séries d'estimation qui découlent des renseignements figurant ci-dessus sur l'hébergement sont associées aux trois variables:

a) Taux moyen d'occupation exprimé selon les trois variables - $A 0(i, j, k)$.

b) Nombre moyen de personnes par chambre/lieu occupé, exprimé selon les trois variables - NP(i,j,k).

c) Nombre total de chambres/lieux - nuits (établissements ouverts) exprimé selon les trois variables - $\mathrm{AR}(\mathrm{i}, \mathrm{j}, \mathrm{k})$.

d) Tarifs moyens d'une chambre pour une personne et pour deux personnes d'une chambre/lieu - nuit (établissements ouverts) exprimé selon les trois variables - PI(i,j,k) et PII(i,j,k).

Grâce a ces renseignements de base, l'estimation du nombre total de journées/nuits de séjour, des dépenses touristiques totales par type d'hébergement, par région, par mois. etc., peut se calculer grâce à une simple opération mathematique. Par exemple: 

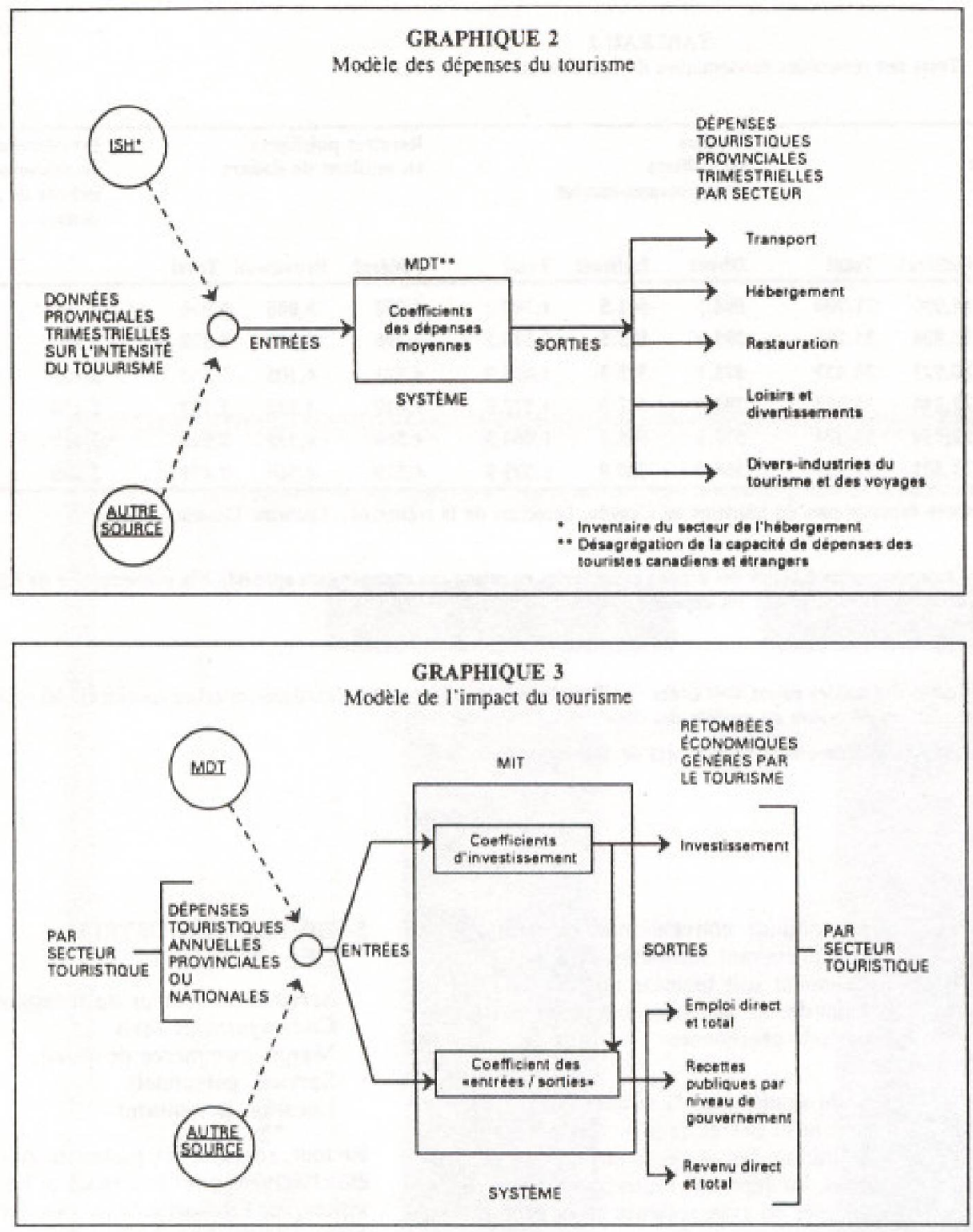

a) Total des dépenses touristiques:

$$
\begin{aligned}
E(i, j, k) & =P(i, j, k) O R(i, j, k) \\
& =A R(i, j, k) P(i, j, k) A O(i, j, k)
\end{aligned}
$$

b) Total des dépenses touristiques potentielles:

$E P(i, j, k)=A R(i, j, k) P(i, j, k)$

c) Total des revenus non réalisés:

$$
\begin{aligned}
& E F(i, j, k)=E P(i, j, k)-E(i, j, k) \\
& =A R(i, j, k) P(i, j, k)(1-A O(i, j, k)) \\
& \text { E }=\text { dépenses touristiques totales } \\
& \mathrm{EP}=\text { total des dépenses touristiques } \\
& \text { potentielles } \\
& \mathrm{EF}=\text { total des revenus non réalisés } \\
& \mathrm{P} \equiv \text { prix } \\
& \text { OR = chambre }- \text { lieu/nuits occupé } \\
& \mathrm{AR}=\text { nombre total de chambres-lieu } \\
& \text { disponible } \\
& \mathrm{AO}=\text { taux d'occupation } \\
& \text { i.j. } \mathrm{k}=\text { les trois variables }
\end{aligned}
$$
tiques concernant l'hébergement, le MDT fournit aussi des estimations sur d'autres dépenses reliées à la restauration, au transport, aux loisirs et aux divertissements, aux divers achats, en se fondant sur le nombre total de journées de séjour - obtenu à partir des données relatives à l'hébergement et des matrices des prix moyens recueillis au cours d'enquêtes sur le tourisme.

Enfin, pour compléter l'estimation des depenses touristiques totales, le MDT ajoute aux dépenses totales effectues par les touristes logeant dans des établissements d'hébergement la partie des dépenses estimées effectuées par les visiteurs qui logent chez des amis ou des parents. Le graphique no 2 présente le mouvement des entrées et des sorties du MDT. Notons que, bien que le principal élément du MDT soit le secteur de l'hébergement (c'est-à-dire que les données relatives à l'hébergement sont utilisées pour estimer le nombre de journées de séjour), les entrées incorporées au MDT peuvent aussi provenir de sources exogènes.

\section{Modèle de I'impact du tourisme (MIT)}

Le but premier du MIT est d'évaluer les retombés économiques des dépenses touristiques sur le plan de la production brute, du revenu, de l'emploi, des recettes publiques et de l'investissement des entreprises.

Contrairement au MDT, le MIT, tout en étant complexe, est très facile à appliquer. Il consiste en une matrice de "coefficients multiplicateurs". Les multiplicateurs utilisés dans le MIT sont définis selon le concept kéneysien: c'est-à-dire les ventes, l'emploi (années-personnes), le revenu, les recettes publiques gênérés par chaque dollar dépensé par les touristes. En appliquant les multiplicateurs aux dépenses touristiques correspondantes, on détermine les retombées economiques du tourisme $e^{(7)}$.

Le modèle d'êvaluation des retombées économiques du tourisme au Canada est unique étant donné qu'il simule les retombées des dépenses touristiques au moyen de modeles structurels d'entrées - sorties de type standard de l'économie nationale et provinciale, et permet d'intégrer toute une série d'équations économétriques dans le système afin d'évaluer les retombées de l'investissement induit par les dépenses initiales du tourisme. La structure des entrées et des sorties du MTT est exposée au graphique $3^{(8)}$.

Mathématiquement, on peut exprimer les relations fonctionnelles généralisées du MIT de la façon suivante:

$$
\begin{aligned}
& \mathrm{I}_{\mathrm{t}}=\alpha_{1} \mathrm{~S}_{\mathrm{t}-1}+\beta_{1} \pi_{\mathrm{t}-1}+\lambda_{1} \mathrm{r}_{\mathrm{t}-1}+\gamma_{1} \mathrm{C}_{\mathrm{t}-1} \\
& \mathrm{I}_{\mathrm{t}}^{\mathrm{P}}=\alpha_{2} \mathrm{~S}_{\mathrm{t}-1}+\beta_{2} \pi_{\mathrm{t}-1}+\lambda_{2} \mathrm{r}_{\mathrm{t}-1}+\gamma_{2} \mathrm{C}_{\mathrm{t}-1} \\
& \mathrm{~T}_{\mathrm{t}}^{\mathrm{T}}=\varsigma_{\mathrm{t}} \mathrm{I}_{\mathrm{t}} \\
& \mathrm{Y}_{\mathrm{t}}^{\mathrm{T}}=\mathrm{f}_{1} \mathrm{E}_{\mathrm{t}}+\mathrm{f}_{2} \mathrm{I}_{\mathrm{t}}^{\mathrm{T}} \\
& \mathrm{G}_{\mathrm{t}}^{\mathrm{T}}=s_{\mathrm{t}}+s_{2} \mathrm{I}_{\mathrm{t}}^{\mathrm{T}} \\
& \mathrm{N}_{\mathrm{t}}^{\mathrm{T}}=v\left(\frac{1}{Z}\right) \mathrm{E}_{\mathrm{t}}+\mathrm{v}_{2}\left(\frac{1}{Z}\right) \mathrm{I}_{\mathrm{t}}^{\mathrm{T}}
\end{aligned}
$$

${ }_{\mathrm{I}}^{\mathrm{P}} \quad=$ investissement total du secteur privé

${ }^{\mathrm{T}}=$ investissement sectoriel provoqué par les dépenses touristiques

$\mathrm{S}=$ ventes sectorielles totales

$\mathrm{Y}=$ revenu total général par les dépenses touristiques

$\mathrm{G}=$ recettes publiques totales genérés par les dépenses touristiques

$\mathrm{N}=$ total des emplois générés par les dépenses touristiques

$\mathrm{P} \quad=$ période

r = taux d'intérêt

$\pi \quad=$ total des bénéfices sectoriels

$\mathrm{C}=$ accélérateur des benéfices sectoriels

$\mathrm{Z}=$ indice de déflation de 1980

$\alpha, \beta, \lambda, \gamma, \varsigma=$ coefficients estimés de façon économétrique

$\mathrm{f}, \mathrm{s}, \mathrm{v}=$ coefficients estimés au moyen de l'analyse des entrées-sorties
$I_{\mathrm{P}}=$ investissement sectoriel total 
TABLEAU 1

Total des retombées économiques du tourisme au Canada 1982-1987

\begin{tabular}{|c|c|c|c|c|c|c|c|c|c|c|c|}
\hline Année & $\begin{array}{l}\text { Recettes } \\
\text { totales } \\
\text { en millions }\end{array}$ & \multicolumn{3}{|c|}{$\begin{array}{l}\text { Revenus } \\
\text { en millions } \\
\text { de dollars }\end{array}$} & \multicolumn{3}{|c|}{$\begin{array}{l}\text { Emploi } \\
\text { en milliers } \\
\text { de personnes-années }\end{array}$} & \multicolumn{3}{|c|}{$\begin{array}{l}\text { Recettes publiques } \\
\text { en millions de dollars }\end{array}$} & $\begin{array}{l}\text { Investissements } \\
\text { en millions } \\
\text { induits de } \\
\text { dollars }\end{array}$ \\
\hline 1982 & 17,669 & 10,527 & 14,030 & 31,700 & 598.5 & 541.5 & $1,140.2$ & 4,092 & 3,888 & 6,936 & 2,790 \\
\hline 1983 & 18,710 & 12,460 & 20,808 & 33,268 & 595.0 & 535.5 & $1,130.5$ & 4,296 & 4,082 & 8,378 & 2,950 \\
\hline $1986^{*}$ & 19,708 & 13,237 & 22,137 & 35,374 & 559.1 & 505.4 & $1,064.5$ & 4,564 & 4,336 & 8,900 & 3,110 \\
\hline 1987 & 20,745 & 13,932 & 23,301 & 37,233 & 564.0 & 509.9 & $1,073.9$ & 4,929 & 4,564 & 9,493 & 3,280 \\
\hline
\end{tabular}

Sources: Le système d'évaluation des retombees économiques du tourisme au Canada, Direction de la recherche, Tourisme Canada.

* Les recettes intérieures de 1986 ne peuvent étre compartes a celles des annétes préédentes en raison des changements apportés à la méthodologie de l"Enquêtte sur les voyages des Canadiens encernant la collecte de données sur les depenses.

Remarques:

- Les données concernant les recettes intérieures des années paires sont tỉrées de l'Enquête sur les voyages des Canadiens, et celles concernant les recettes intérieures des années impaires sont une estimation découlant du modèle des dépenses du tourisme (MDT).

- Les donnés concernant les recettes toursitiques internationales proviennent de Statistique Canada.

La décision d'intégrer les fonctions d'investissement au MIT était fondée sur le fait que l'investissement constitue une grande proportion de la demande finale et qu'il serait erroné d'évaluer la performance des provinces canadiennes sans tenir compte du facteur d'investissement, étant donné les variations qui les caractérisent au chapitre de la consommation, de l'épargne et de l'investissement.

La théorie économique sous-jacente aux équations d'investissement du MIT repose sur l'hypothèse que l'investissement à la période " $t$ " est une fonction linéaire d'un groupe de facteurs (ventes, bénéfices, taux d'intérêt et propension à investir) appelé "accélérateur", dans la période "t-1".

$$
\begin{gathered}
C_{t}=\left(\frac{I_{t}-I_{t-1}}{I_{t-1}}\right) 100 \\
I_{t+1}=v_{t} \pi_{t}+\gamma_{t}\left(\frac{I_{t}-I_{t-1}}{I_{t-1}}\right) ; v>0>\lambda \geqslant 0
\end{gathered}
$$

La décision d'investir prise au moment " $\mathrm{t}$ ", est fondée sur la rentabilité du secteur à ce moment-là et de l'activité d'investissement sectoriel cyclique qui a lieu au moment " $\mathrm{t}$ "; l'investissement prend un certain temps pour se concrétiser après que la décision d'investir est prise. L'accélérateur " " $\mathrm{C}$ " représenté l'état cyclique de l'économic sectorielle. Exprime comme taux de changement, l'accélérateur reflète l'argument macro- économique conventionnel voulant que le niveau courant absolu des dépenses d'investissement soit fonction du taux de changement de ces dépenses enregistré pendant la période précédente.

Étant donné que la simulation du modèle structurel des entrées-sorties nécessite une définition des secteurs et produits touristiques, les dépenses touristiques ont été désagrégées en cinq secteurs et 17 produits, de la façon suivante ${ }^{(9)}$ :

\section{1- TRANSPORT}

avion

bateau

chemin de fer

autocar (interurbain et rural)

autoroute, péage et entretien des ponts, commerce de détail (station-service, concessionnaire $d^{+} u n e$ marque d'automobiles et atelier de réparation)

\section{2- HÉBERGEMENT}

Service d'hébergement

\section{3- RESTAURATION}

Repas
Marge de service sur les boissons alcoo-
lisées

\section{4- LOISIRS ET DIVERTISSEMENTS}

Cinéma et divertissement Voyages et divertissements Autres services récréatifs

\section{5- DIVERS - INDUSTRIE DES VOYAGES}

Services au secteur du transport Compagnies de taxis

Marge, commerce de détail

Services personnels

Location de voitures

En tout, soixante-six passes de machine ont été effectuées pour le Canada et les dix provinces, sur l'industrie du tourisme et ses cinq sous-secteurs. Les résultats de chaque simulation comprennent les coefficients multiplicateurs concernant les ventes totales, le revenu, l'emploi, les recettes publiques et les investissements d'entreprises. Ces multiplicateurs peuvent être facilement utilisés pour calculer les retombées économiques du tourisme. Il s'agit d'une simple multiplication de deux chiffres, celui des dépenses touristiques et du coefficient multiplicateur approprié.

\section{Les sorties du MIT}

L'ampleur et l'importance des contributions touristiques à l'économie canadienne ont été définies pour la première fois pour l'année 1971 au moyen d'un modèle économétrique, élaboré par le présent auteur, des données sur les dépenses touristiques émanant de Statistique Canada et de l'enquéte repère de 1971 sur les voyages intérieurs des Canadiens ${ }^{100}$.

En ce qui concerne les années allant de 1972 à 1978 inclusivement, les retombées économiques du tourisme au Canada ont été simulées au moyen du MDT/MIT intégré: les dépenses touristiques ont été estimées au 
moyen du MDT en se fondant sur les données obtenues auprès du secteur de l'hébergement, les ventes, le revenu, l'emploi, les recettes publiques at les investissements d'entreprises générés ont êté simulés avec le MIT.

Lorsque I'Enquêtte sur les voyages des Canadiens a ếtế reprise sous sa forme complète en 1979, elle fournit les détails des dépenses touristiques intérieures pour les années 1979 et 1980. On procéda au passage machine du MIT et les retombées économiques du tourisme pour cette année furent calculées sans l'aide du MDT; les dépenses touristiques internationales furent obtenues auprès de Statistique Canada, et les dépenses touristiques des Canadiens au Canada, furent extraites de l'Enquête sur les voyages des Canadiens. Depuis 1980, le programme de recherche de Tourisme Canada prévoit l'exécution d'une enquête biennale sur les voyages des Canadiens toutes les années paires. La simulation du MDT était nécessaire durant les années impaires pour calculer les données sur les dépenses touristiques des Canadiens utilisées comme entrées pour le MIT. Le graphique 1 contient les sorties finales du MDT/MIT concernant les retombées économiques pour les années 1982 à 1987. Ces données représentent les statistiques officielles des retombées économiques du tourisme au Canada.

\section{Usages du MDT/MIT}

Le MTD/MIT est le premier et le seul modèle macro-économique intégré ayant trait au tourisme canadien. Il constitue le seul modèle économique fournissant des données complètes, cohérentes, compatibles et comparables sur les retombées économiques du tourisme dans tout le Canada. Le MDT/MIT a été élaboré du moment où le besoin s'en faisait le plus sentir, durant la métamorphose de l'industrie canadienne du tourisme. Il a rendu possible l'élaboration du premier programme de développement de l'industrie touristique au Canada (PDIT). Il a aussi servi à la conception des ententes auxiliaires fédérales-provinciales ayant trait à l'indus" trie du tourisme. L'utilisation du MDT/MIT est très répandue. Il simule des options ayant trait à la planification de politiques et de programmes, aide à établir des scénarios visant ă résoudre des problèmes de développement et de commercialisation. Il sert aussi à évaluer des propositions de projets de développement ou à établir un plan de commercialisation à court terme.

On s'attend a ce que le MDT/MIT devienne encore plus efficace lorsque le compte satellite du tourisme sera complètement opérationnel et que tous les secteurs de l'industrie seront définis, regroupés et intégrés au Système de comptabilité nationale.

\section{Évaluation du MDT/MIT}

Depuis sa création en 1973, le MDT/MIT a évolué: de logiciel applicable au niveau national, il est devenu un système macro écono- mique transcanadien interprovincial; de logiciel de macro-ordinateur de la première génération en FORTRAN, il est devenu un logiciel de micro-ordinateur de bureau convivial (11). Il a également suscité l'élaboration d'un modèle-prototype servant à mesurer les retombées économiques du tourisme au niv. eau régional et municipal ${ }^{(12)}$.

Les concepts et la structure du MDT/MIT ont été examinés rigoureusement par des économistes de mềme que par des spécialistes de l'industrie. Il a été présenté au cours de réunions de sociétés savantes et d'organisations internationales telles que l"Organisation mondiale du tourisme, 1'Organisation de coopération et de développement économiques, et la Commission de la communauté européenne. Il a été discuté au cours de conférences de spécialistes telles que l'American Statistical Association, la Société canadienne de recherche opérationnelle, l'Association canadienne et internationale de recherche sur le tourisme (Canadian and International Travel and Tourism Association) et le Comité fédéral-provincial de recherche sur le tourisme $^{(13 .}{ }^{14)}$. Il a fait l'objet d'articles dans des revues professionnelles et touristiques. Mais le plus important verdict a été rendu par les membres de l'industrie canadienne du tourisme: ils ont accepté et adopté le MDT/MIT. $f$

\section{Notes explicatives}

(1) L'Otfice du Tourisme du Canada, Assessment of Alternate Approaches the Development of a Tourism Impact Model, rapport préparé par le Bureau des conseillers en gestion, ASC, 1974.

(2) Frechtling Douglas C., U.S. Travel Data Center, Assessing the Impacts of Travel and Tourism, Measuring Economic Benefits. Travel, Tourism and Hospitality Research, Edited by Ritchie, J.R.B. and Goeldner, C.R.. 1987.

(3) Division of Tourism (1974). Tourism Employment Study, Sacramento, Californie, Département du commerce.

(4) West Virginia University (1981), Creating Economic Growth and Jobs through Travel and Tourism. Washington, D.C.

(5) Office du tourisme du Canada, 1975. Tourism Expenditures Model: A Functional Planning and Policy-Making Tood, rapport préparé par le Bureau des conseillers en gestion, ASC.

(6) Fritz, Richard G., Konecny, Michael F, and Stoucas, Panayotis, D. Tourism Expenditures Model: A Functional Planning and Policy-Making Tool, Regional Science and Urban Economics, février 1979.

Q) Archer, Brian, 1977, Input-output Analysis: Its Strenghts, Limitations and Wanknesses. Eight Annual Conference Proceedings, Salt Lake City. the TTRA.

......... 1977 , Tourism Multipliers, The State of the Art. Bangor, Wales: University of Wales Press.

........ 1982, Valve of Multipliers and Their Poli. cy Implications. University of Surrey, Guilford, U.K.

(8) Otfice du tourisme du Canada, 1975, Tourtsm Eco. nomic Impact Model, Rapport préparé par le Bureau des conseillers en gestion, ASC.

(9) Chau, Peter H.N., 1982, Tourism Within the Input-Output Structural Model, document de travail interne. Direction de la recherche. Tourisme Canada.
(10) Ótfice du Tourisme du Canada, 1974, Tourisme: son ampleur et son importance. Bulletin de racherche no 2, Canada.

(11) Chau, Peter H.N., 1996, Tourism Impact System: A Situation Report, Direction de la recherche. Tourisme Canada.

(12) Tourisme Canada, 1988, Initiative de dbveleppe ment de I'industrie touristique dans les collect:vites: évaluation des retombóes fconomiques: du tourisme Niagara Falls, Direction de la recherche. Tourisme Canada.

(13) Chau, Peter H.N., 1976, Unification of International Tourism Statistics, expose de principe, preparé a l'intention du colloque de l'Organisation mondiale du tourisme sur la normalisation des Sta: tistiques du tourisme international, Caracas. Venezuela.

(14) Taylor, Gordon de R and Doctoroff, Mark, Máthode de Systàme Intégré de Próvision pour un Office National du Tourisme, Communication présentée au Colloque sur les perspectivas d'aven. nir du tourisme ainsi que le tourisme et la balance des paiements, Haslemere, Surrey. Royaume-Uni, 1974. 